\title{
2 APRENDIZAJE COOPERATIVO: BASES TEÓRICAS Y HALLAZGOS EMPÍRICOS QUE EXPLICAN SU EFICACIA
}

\author{
Edurne Goikoetxea y Gema Pascual
}

Universidad de Deusto

Un desafío permanente para las organizaciones educativas, y sin duda para los profesores, es encontrar formas de organizar y conducir la instrucción en el aula que concilien dos objetivos: maximizar el aprendizaje y educar personas capaces de cooperar y de establecer buenas relaciones humanas. El aprendizaje cooperativo (AC de aquí en adelante), tema del presente trabajo, puede contribuir directamente a llevar a buen término este desafío.

El AC es un término genérico para referirse a numerosas técnicas de organizar y conducir la instrucción en el aula caracterizadas por el trabajo en grupos pequeños (usualmente 4 a 5 miembros) de alumnos heterogéneos (bajo, medio y alto rendimiento; varones y mujeres; etnias diferentes) para lograr objetivos comunes de aprendizaje (véase e.g., Slavin, 1990; Johnson, \& Johnson, 1994). A diferencia del llamado "aprendizaje colaborativo", categoría más amplia que cobija formas de colaboración entre estudiantes no necesariamente estructuradas, incluso informales, el AC tiene lugar cuando existe una estructura definida de la situación de aprendizaje. De hecho, una idea transmitida por muchos autores al definir el AC es que se trata de algo más que de una forma de agrupamiento de los estudiantes. Siguiendo a Slavin (1983), se trata más bien de un arreglo de la situación de aprendizaje que incluye una "estructura cooperativa de la tarea" y una "estructura cooperativa de los incentivos» (p. 431). Por una parte, en el AC la tarea es común y, por tanto, se permite o se exige a los alumnos que trabajen juntos en ella, coordi- 
nando sus esfuerzos para llevarla a buen fin. Por otra parte, los estudiantes suelen depender unos de otros para recibir el incentivo o recompensa, que puede ser: grupal basada en el rendimiento grupal, grupal basada en el aprendizaje individual, e individual. Por tanto, aunque todas las técnicas de AC incluyen una estructura cooperativa de la tarea, no todas cuentan con una estructura cooperativa de incentivo.

La investigación sobre los efectos del AC ha encontrado consistentemente que estas técnicas mejoran las relaciones humanas en grupos heterogéneos debido a diferencias étnicas (e.g., DeVries, \& Edwards, 1974; Slavin, 1978b, 1979; Slavin, \& Cooper, 1999) y a diferencias en aptitudes mentales o físicas (e.g., Madden, \& Slavin, 1983; Slavin, 1990). También han encontrado que promueven el logro cognitivo y el rendimiento académico, al menos en comparación con métodos competitivos (dos o más individuos se comparan entre sí y se recompensa al de mayor rendimiento, o bien de acuerdo a un criterio) e individualistas (cada individuo es recompensado según su propio rendimiento, con independencia del rendimiento de los demás). Una de las razones es porque ofrecen a los estudiantes «mayor oportunidad para discutir, para aprender unos de otros y para alentar la excelencia entre ellos" (Slavin, \& Cooper, 1999, p. 648). Más adelante se discute la evidencia sobre los requisitos necesarios para lograr estos resultados. Asimismo, aunque no de forma tan consistente, los estudios han encontrado que los métodos de $\mathrm{AC}$ mejoran el estatus social y la conducta de estudiantes en desventaja por razones diversas (e.g., minusvalías físicas), aunque no siempre consiguen promover lazos de amistad (Madden, \& Slavin, 1983); previenen problemas como las reacciones negativas ante la integración y la diversidad, el egocentrismo o la falta de conductas prosociales; y promueven el locus de control interno, el altruismo y las habilidades necesarias para ser un buen ciudadano (e.g., Adams, \& Hamm, 1990; Cohen, 1994; Johnson, \& Johnson, 1994; Slavin, 1990).

Es probable que esta inusual combinación de beneficios intelectuales y actitudinales, junto con su versatilidad, haya convertido al $\mathrm{AC}$ en un procedimiento educativo ampliamente investigado a lo largo de las tres últimas décadas (Johnson, Johnson, \& Stanne, 2000) y crecientemente aplicado en todos los niveles de la educación, desde infantil hasta universitario; en todas las áreas de contenido, desde lengua hasta ingeniería; y en situaciones tradicionales y no-tradicionales (Johnson et al., 2000). De hecho, aunque todavía hoy es menos empleado que otras formas tradicionales de conducir el aprendizaje (7 a 20\% del tiempo de clase; Johnson, \& Johnson, 1994), el AC está siendo utilizado de forma creciente y sostenida, al menos entre los profesores norteamericanos (79\% de los profesores de primaria y $62 \%$ de los de secundaria; Slavin, 1995b).

El estudio de revisión más reciente sobre el AC (Johnson et al., 2000) destaca tres razones que contribuyen al creciente uso del AC: la amplia evidencia empírica sobre su eficacia, su variedad (desde altamente concretos y prescriptivos hasta conceptuales y flexibles) y su fundamentación en diversas 
teorías (desde antropológicas, sociológicas y económicas hasta psicológicas). No obstante, en la actualidad se encuentra que la fundamentación y los efectos de estos métodos no siempre son bien conocidos, incluso por quienes los utilizan, a pesar de ser éste un conocimiento importante para una aplicación sostenida y fundada de estos métodos por parte del profesorado.

Sería un empeño imposible resumir aquí la literatura y la investigación existente sobre AC y, además, en español hay obras de referencia sobre este tema (e.g., García, Traver, \& Candela, 2001; Johnson, Johnson y Holubec, 1995; Lobato, 1998; Ovejero, 1990; Serrano, González-Herrero, \& Martínez-Artero, 1997; véase también Domingo, \& Giraldo, 2002; Goikoetxea, 1990). Nuestro objetivo es más modesto, y se ciñe, primero, a revisar algunas de las bases teóricas que sustentan la eficacia del AC; segundo, a describir brevemente los métodos de $\mathrm{AC}$ que han recibido mayor respaldo empírico; y tercero, a resumir los resultados más importantes de los estudios de revisión y de meta-análisis sobre los efectos del AC y los mecanismos responsables de dichos efectos. Por tanto, al preparar este trabajo hemos tenido en cuenta no sólo la necesidad de extender las innovaciones educativas que cuentan con evidencia empírica sobre sus resultados, sino la convicción de que la puesta en marcha cabal y el mantenimiento de nuevas prácticas educativas exige al profesional conocer los fundamentos teóricos y los resultados sobre sus efectos.

\section{BASES TEÓRICAS DEL AC}

\section{EL AC Y LA MEJORA DE LAS RELACIONES INTERÉTNICAS}

Como ya se dijo previamente, los efectos del AC en la mejora de las relaciones interétnicas han sido ampliamente estudiados desde hace casi tres décadas (e.g., DeVries, \& Edwards, 1974; Slavin, 1978b, 1979; Slavin, \& Cooper, 1999). La mayoría de los estudios han empleado indicadores sociométricos para medir el cambio en las relaciones intergrupales, además de medidas observacionales, medidas actitudinales y autoinformes.

Este apartado se centra, pues, en revisar la base teórica que sustenta los beneficios del AC en las relaciones interétnicas. Aunque estos beneficios no son los únicos, aquí se hace hincapié en estos porque forman parte de los fines originarios de estos métodos y porque la diversidad cultural es una realidad creciente en las aulas españolas, con escasa tradición, sin embargo, en la educación de estudiantes de distintas culturas y religiones (véase Grant, \& Sleeter, 1986 para una revisión de la necesidad de integrar la influencia simultánea de la clase social, la etnia y el género en la conducta social de la escuela). Por otra parte, a comienzos del siglo XXI no cabe preguntarse si la integración de estudiantes de distintas culturas y religiones es deseable, por 
obvias razones morales y fácticas. Pero sí es preciso responder a preguntas sobre qué modos son los más idóneos para llevar adelante una buena integración.

Para comprender por qué el $\mathrm{AC}$ mejora las relaciones interétnicas, una referencia ineludible es la obra clásica «Nature of prejudice» de Allport (1954/1962). En ella, Allport evalúa la experiencia acumulada acerca de los efectos de la integración de minorías en las relaciones interraciales en distintos ámbitos, con el fin de extraer un conjunto de principios que expliquen cuándo el contacto interracial conduce a establecer buenas relaciones. Un dato preocupante de este trabajo fue que el mero contacto superficial no sólo no mejoraba las relaciones interraciales, sino que podía empeorarlas, especialmente si el contacto era competitivo y entre individuos de estatus social muy diferente. Allport señala, por tanto, que la convivencia de diversos grupos raciales en un mismo lugar, ya sea el vecindario, el trabajo o el aula, es compleja y potencialmente conflictiva, aunque los individuos tengan buena voluntad. No es el mero hecho de vivir juntos, sino la comunicación y el contacto lo que importan para el establecimiento de buenas relaciones humanas. Estudios posteriores vuelven a mostrar el reducido efecto de la integración per se en las relaciones interraciales (e.g., Stephan, 1978) y siguen debatiendo el reto de la integración (e.g., Khmelkov, \& Hallinan, 1999).

Sin embargo, Allport también encuentra estudios sobre el efecto de situaciones de contacto donde individuos de diferentes etnias trabajan de igual a igual en grupos para alcanzar objetivos comunes y tienen oportunidades de conocerse unos a otros como individuos. Cuando ocurren estas circunstancias, Allport observa que los miembros de tales grupos establecen lazos de amistad y abandonan parte de los prejuicios de unos hacia otros. Estos datos dan origen a la llamada "teoría del contacto», que ha dominado la investigación en las ciencias sociales sobre las relaciones interétnicas a lo largo de casi cinco décadas. El propio Allport resume la esencia de la teoría del contacto así:

El prejuicio (salvo que esté firmemente enraizado en la estructura de carácter del individuo) puede ser reducido por un contacto a igual estatus entre los grupos mayoritarios y minoritarios, en procura de objetivos comunes. El efecto se ve muy reforzado si este contacto está sancionado por soportes institucionales (es decir, por la ley, la costumbre o la atmósfera local), y siempre que sea de tal suerte que lleve a percibir intereses comunes y una común humanidad en los miembros de los dos grupos (p. 309).

La hipótesis de contacto predice, pues, que habrá un cambio favorable en las actitudes y la atracción interpersonal, si el contacto personal entre miembros de grupos distintos ocurre en las siguientes cuatro condiciones: Primera, la situación de contacto debe estimular una relación de interdepedencia o de cooperación para alcanzar un objetivo conjunto. Segunda, las normas sociales de la situación de contacto deben favorecer el concepto de igualdad 
de grupos y la relación igualitaria entre ellos. Tercera, la situación de contacto debe tener un gran potencial de conocimiento de forma que promueva una relación que revele suficientes detalles sobre los miembros del «otro» grupo, como para verlos como individuos y no sólo como personas con características del grupo estereotipado. Cuarta, el estatus de los participantes de los dos grupos debe ser igual en la situación de contacto.

Cómo satisface el AC los criterios de la teoría del contacto. Las razones que apoyan el uso del AC en la mejora de las relaciones interétnicas provienen de la satisfacción de los criterios de Allport (1954/1962). A continuación se desarrolla con más detalle esta cuestión.

Primero, el AC ofrece una meta común. Allport (1954/1962) sostiene que «solamente el tipo de contacto que hace que la gente realice cosas en conjunto tenderá a producir un cambio en las actitudes» (p. 305). Este principio puede aplicarse claramente a los métodos de AC, pues ofrecen una tarea $\mathrm{u}$ objetivo común y generalmente incentivos también comunes. Se espera que la meta común que el grupo ha de alcanzar se convierta en lo más importante, dirigiendo la atención hacia la tarea y desplazándola de las características étnicas de los miembros. En este sentido, el esfuerzo conjunto por resolver la tarea hace las veces de "punto de encuentro" entre miembros de diferentes minorías y se espera que la actividad cooperativa para lograr esa meta genere solidaridad grupal.

Segundo, la puesta en práctica del AC comunica apoyo institucional a las relaciones interétnicas. Allport (1954/1962) supone que el contacto interétnico mejora las relaciones si las instituciones en las que ocurre éste apoyan claramente la igualdad y la interacción interétnica. La razón es que la mayoría de las personas tienen sentimientos conflictivos respecto a la integración. Por una parte, se sienten incómodos al interactuar con otros diferentes, por otra, se avergüenzan de estos sentimientos debido a que mantienen como valor la igualdad de las personas. Por tanto, las personas de grupos mayoritarios aceptarán mejor la interrelación si no tienen que iniciarla ellas mismas, sino sólo aceptar el contacto impulsado por normas institucionales (p. 304). Y aunque, como señala Allport, se puede suponer que en la escuela existe este apoyo institucional, en la realidad son pocas las que experimentan con métodos que favorecen las relaciones interétnicas, ya sea por desconocimiento por parte de los profesores sobre qué hacer, o por la idea de que, mejorando el rendimiento de los alumnos de etnias minoritarias y presionando para que adopten hábitos de la mayoría, los problemas de interrelación desaparecerán. Sin embargo, el rendimiento no es una variable que se modifique fácilmente y la asimilación forzosa tampoco ha probado ser eficaz. Mientras, los alumnos dejan de recibir mensajes que comunican el valor de la interrelación entre ellos. Precisamente uno de los cambios que introducen los métodos de AC en escuelas multiculturales es que legitiman el contacto interétnico y lo juzgan positivo (Slavin, 1983). Los alumnos pueden sentirse apoyados si los profesores estimulan la interacción interétnica y 
catalogan este tipo de contacto como normal y deseable. Este clima se puede crear sin necesidad de decir ni una sola palabra, la conducta del profesor ya indica que aprueba el contacto.

Tercero, el AC da oportunidad de mantener un contacto más profundo con otros, generando así una percepción de semejanza o naturaleza humana común. Las oportunidades para discutir y para aprender unos de otros permiten el conocimiento del "otro" como individuo y no sólo como persona con características del grupo estereotipado. Aunque Allport (1954/1962) llega a la conclusión de que «el conocimiento y las relaciones con miembros de grupos minoritarios favorecen las actitudes tolerantes y amistosas» (p. 296), la relación entre estas dos variables no es de ninguna manera perfecta. Por tanto, Allport indica, de forma más prudente, que "los contactos que redundan en una intensificación del conocimiento y de las relaciones tienden a engendrar creencias más auténticas acerca de los grupos minoritarios, y por esta razón contribuyen a la reducción del prejuicio" (p. 296).

Cuarto, el criterio de igualdad de estatus, necesario, pero no suficiente, para que el contacto sea positivo según Allport (1954/1962), exige que éste ocurra entre individuos de semejante estatus. Éste es el principio más costoso de satisfacer en los métodos de AC, porque el estatus de los individuos de ciertas etnias (o con ciertas características intelectuales o físicas) puede ser bajo. Sin embargo, puede aplicarse a la situación de clase, mejor que a otros escenarios, pues los estudiantes de un mismo curso mantienen un estatus semejante con independencia de su etnia, sexo o nivel de rendimiento. Además, la asignación de los estudiantes a grupos de AC da una cierta identidad común de inicio. Los alumnos se encuentran en un marco de trabajo que facilita, aunque no garantiza, la igualdad de estatus, debido a su pertenencia a un mismo grupo. Por otra parte, el logro de esta condición se favorece si los métodos de AC adoptan una estructura de incentivo que recompensa al grupo en base al progreso individual de cada miembro, de tal forma que todos puedan aportar ganancias a su grupo. También cuando el AC se utiliza en áreas en las que los alumnos de estatus más bajo sobresalen o al menos tienen un rendimiento semejante a los demás (Slavin, \& Madden, 1979; Aronson, \& Bridgeman, 1979).

El AC y la mejora del rendimiento académico. Para comprender por qué el AC mejora el rendimiento académico, sería preciso remitirse a perspectivas motivacionales, de cohesión social, cognitivas y evolutivas, tratadas en varios trabajos (véase e.g., Slavin, 1990, 1995b). De hecho, estas razones son las más discutidas todavía en la literatura, y no existe una única perspectiva teórica. En el siguiente apartado, dadas las limitaciones de espacio, no se aborda este aspecto con la debida profundidad, pero se revisa uno de los modelos de instrucción eficaz que apoya los beneficios académicos de los métodos de AC y los sitúa como complementos y alternativas reales a los métodos tradicionales. 
A menudo, ofrecer una instrucción eficaz para todos los estudiantes de una misma clase, cada uno con sus diferentes conocimientos, habilidades y motivaciones, es difícil y supone un "caballo de batalla" para el profesor. En respuesta a este problema, se han organizado distintos modos de agrupamiento cada uno con ventajas y desventajas (véase e.g., Slavin, 1987b). El AC es uno de ellos y parece ofrecer una enseñanza eficaz, sin perder oportunidades de integración y contacto entre diferentes alumnos. De hecho, como ya se dijo en el apartado anterior, la investigación muestra que estos métodos producen un rendimiento y una productividad superior a otros métodos tradicionales. Estos efectos se han operativizado en variables tales como las calificaciones escolares, el rendimiento en tests aptitudinales, y el rendimiento en medidas tan variadas como el razonamiento de nivel superior, la creación de nuevas ideas y la transferencia del aprendizaje (e.g., Johnson, \& Johnson, 1994; Slavin, 1983).

Para describir el AC en términos de las variables de la instrucción eficaz, vamos a revisar el modelo de enseñanza eficaz de Slavin (1984) que es, a su vez, una extensión del influyente modelo de aprendizaje escolar de John Carroll de 1963 (citado en Slavin, 1984, 1987a). Carroll propone cinco elementos que contribuyen a la eficacia de la instrucción. Los tres primeros, las aptitudes, la habilidad para entender la enseñanza y la perseverancia, son los elementos más difíciles de cambiar, pues son características del estudiante. Los dos restantes, la calidad de la instrucción y la cantidad de tiempo de enseñanza, son los elementos alterables, porque están bajo el control del profesor. Precisamente el modelo de Slavin parte de estos elementos alterables e incluye cuatro componentes (véase también Slavin, 1987a, 1987b, 1995a): Primero, la calidad de la instrucción o grado en que la presentación de los conceptos y habilidades facilita el aprendizaje significativo y el grado en que el profesor ofrece respuestas. Segundo, el nivel de instrucción apropiado o grado en que el profesor asegura que los estudiantes están preparados para aprender una nueva lección. Tercero, el estímulo o grado en que el profesor asegura la motivación de los estudiantes para trabajar en las tareas y para aprender los contenidos. Cuarto, el tiempo o grado en que los estudiantes disponen del suficiente tiempo para aprender los contenidos tratados. La variable tiempo se divide, a su vez, en dos categorías: tiempo dedicado o tiempo empleado en la instrucción, cuyo control está en el profesor, y tiempo invertido o tiempo que invierte el alumno en el aprendizaje activo, cuyo control está en el alumno.

Según Slavin (1984), el desarrollo de una instrucción eficaz depende de que estos cuatro elementos sean adecuados, no basta que sólo uno de ellos sea efectivo si los restantes son débiles. De hecho, puesto que la relación entre los elementos y el rendimiento escolar es multiplicativa, si uno de los elementos es cero, el rendimiento es cero; de ahí que sea más recomendable conseguir un nivel moderado en los cuatro elementos, que uno excelente en uno sólo de ellos (Slavin, 1987a, p. 99). Por ejemplo, la enseñanza programada es fuerte en uno de estos elementos (adaptación al nivel del estudiante), 
pero débil en los otros tres. Esto explica por qué la investigación no ha mostrado beneficios importantes en el rendimiento académico con este tipo de enseñanza, a pesar de ser una enseñanza con suficiente base teórica y con materiales muy bien diseñados (Slavin, 1987b). Otro ejemplo son los agrupamientos homogéneos en función del rendimiento o de la habilidad. Éstos también son fuertes en la adaptación al nivel del estudiante, pero débiles en el incentivo (e.g., expectativas bajas sobre el potencial académico de los estudiantes por parte del profesor y de los propios estudiantes) y, como consecuencia también, en el tiempo dedicado al estudio, razones por las que tampoco han mostrado beneficios importantes en el rendimiento.

Cómo satisface el AC los elementos de la enseñanza eficaz. Las razones que apoyan el uso del AC como alternativa válida para organizar la enseñanza provienen, en parte, de la satisfacción de los elementos de Slavin (1984) sobre la enseñanza eficaz. Esta cuestión se desarrolla con más detalle a continuación.

Primero, el criterio de la calidad de la instrucción puede aplicarse plenamente al AC, puesto que atiende los aspectos destacados por Slavin para el logro de una enseñanza de calidad. Por un lado, satisface aspectos relacionados con la presentación de las lecciones, dado que en muchos métodos de AC es el profesor quien sigue explicando los contenidos bajo estudio y, en todos, es quien planea tareas que garantizan que el estudiante encuentre sentido a los nuevos contenidos. Por otro, satisface sobradamente aspectos relacionados con la cantidad de enseñanza directa y la evaluación continua, pues libera al profesor para realizarlas y admite la colaboración de los estudiantes en estas funciones tradicionalmente asignadas sólo al profesor (véase también Creemers, \& Sheerens, 1997 para una revisión sobre la importancia de la evaluación en el logro de niveles superiores de aprendizaje).

Segundo, el criterio del nivel apropiado de instrucción es quizá el problema más difícil de satisfacer para la organización escolar, y de ahí que hayan surgido, como antes se veía, varias formas de agrupamiento. Algunos métodos de AC representan una forma efectiva de acomodación a las diferencias individuales al liberar tiempo al profesor para dedicarlo a la enseñanza de grupos con niveles similares de conocimiento u homogéneos (véase e.g., Slavin, Leavey, \& Madden, 1984; Slavin, Madden, \& Leavey, 1984).

Tercero, respecto al estímulo, el AC es un método que asegura la motivación extrínseca del alumno a través de diversos tipos de incentivos externos como premios, calificaciones o diplomas, tanto a nivel individual como a nivel grupal (véase también O'Donnell, 1996, para un estudio sobre los efectos positivos de los incentivos explícitos en la motivación). Además, también asegura la motivación intrínseca, ampliamente estudiada desde las perspectivas motivacionales, pudiéndose pensar que los métodos de AC son atractivos en sí mismos (Sharan, \& Shachar, 1988). En concreto, la interacción entre los miembros del grupo, el trabajo para alcanzar un objetivo común y 
el mismo sistema de recompensas empleado por los métodos de AC generan en los alumnos incentivos internos tales como el deseo de aumentar su rendimiento para contribuir al éxito del grupo, el interés por ayudar a los compañeros más retrasados o la curiosidad intelectual. También la posibilidad de crear relaciones estables y perdurables que ofrecen estos métodos pueden generar en los alumnos el interés por trabajar arduamente (véase Johnson et al., 1995, pp. 59 y ss.).

Cuarto, el tiempo que se dedica a aprender, tanto el dedicado como el invertido, parece ser atendido más fácilmente desde estructuras cooperativas que desde otro tipo de estructuras. Por ejemplo, más de 30 estudios han mostrado que el AC favorece que el estudiante dedique más tiempo y más intensidad a las tareas frente a otras estructuras (Johnson, \& Johnson, 1994). Una razón que puede explicar este efecto reside en que el AC promueve la atención a la tarea y la motivación y éstas son variables que se han encontrado relacionadas con el tiempo dedicado al aprendizaje (e.g., Slavin, 1990).

\section{MÉTODOS DE AC CON EVIDENCIA EMPÍRICA SOBRE SUS EFECTOS}

Antes de abordar los resultados de los estudios de revisión sobre los efectos de los métodos y los mecanismos que los explican, se describen los nueve métodos de AC mejor investigados y cuyos estudios permiten calcular el tamaño del efecto (Johnson et al., 2000; Slavin, \& Cooper, 1999). Todos, salvo dos, han sido descritos por Ovejero (1990) y por Slavin (1990); no obstante, ofrecemos también otras referencias bibliográficas para el lector interesado. Este apartado trata de ser un análisis breve de cómo cada método atiende algunas variables básicas (i.e. tamaño de los equipos, funciones del profesor) y algunas variables que, según los estudios de revisión (Slavin, 1983), más contribuyen a la eficacia de los métodos (i.e. tarea y recompensa).

\section{Aprender Juntos (Learning Together; Johnson, \& Johnson,} 1994). Es un método aplicable a una extensa variedad de materias. Los alumnos trabajan en equipos heterogéneos de 4-6 miembros. El profesor enseña la lección a toda la clase, como en los métodos tradicionales, y deja tiempo para el trabajo en equipo. El objetivo es que todos los miembros del equipo dominen la lección presentada y ayuden a sus compañeros en el aprendizaje de la misma. La tarea es grupal. Todos los miembros del grupo trabajan juntos para completar un material de trabajo único. Este material, compuesto por diversos ejercicios sobre la lección y sus soluciones, además de ser la base para la evaluación del grupo, sirve a los alumnos para practicar, ayudarse unos a otros, evaluarse a sí mismos y evaluar a sus compañeros. La recompensa es grupal en base a la calidad del material presentado, el rendimiento en un test $u$ otros productos, pero siempre grupales. 
Este método es el menos complejo de los métodos de AC y el más cercano al modelo puro cooperativo, en comparación con otros métodos que contienen elementos más individualistas o competitivos. Pero tiene un gran inconveniente, y es que no permite conocer la contribución de cada miembro al grupo, con lo que ello significa en el detrimento de la responsabilidad individual. En Johnson, Johnson, Holubec, y Roy (1984) se ofrecen orientaciones prácticas para llevar a cabo esta estrategia.

2. Grupo de Investigación (Group Investigation; Sharan, \& Sharan, 1992). Es un método aplicable a variedad de materias y de gran utilidad para especializar a los alumnos en una tarea. Los alumnos forman sus propios equipos de 2-6 miembros. El profesor no da la clase como en los métodos tradicionales, aunque puntualmente puede hacerlo. Sus principales funciones son facilitar recursos y supervisar el trabajo de los grupos de investigación, así como entrenar y modelar habilidades de comunicación. La tarea es grupal y consiste en hacer un informe grupal sobre un tema. Cada equipo, según sus intereses o conocimientos previos, elige un tema de una unidad que debe estudiar toda la clase. Para asegurar la responsabilidad individual, cada tema se subdivide en tantas partes como miembros tiene el equipo y cada miembro ha de responder por ella. No obstante, al final, todos los miembros del equipo han de coordinarse para llevar a cabo las distintas actividades que el proyecto de investigación exige: buscar información, evaluarla, sintetizarla, preparar el informe final del grupo y presentar dicho informe al resto de la clase. En este método han sido probadas recompensas de varios tipos, desde grupales (i.e. calidad del informe y de la presentación) hasta individuales (i.e. examen individual sobre los contenidos expuestos por todos los equipos). No obstante, los autores de esta técnica rechazan los sistemas de recompensa tangibles y abogan por no dar nada, excepto la oportunidad de continuar trabajando en este tipo de grupos, confiando en el atractivo de los métodos de AC en sí mismos (Sharan, \& Shachar, 1988).

Este método ha sido desarrollado recientemente por Shlomo Sharan, Yael Sharan y Rachel Hertz-Lazarowitz en Israel. No obstante, las raíces del mismo se encuentran en John Dewey (1859-1952). Asimismo, se trata de un método adecuado para trabajar "tareas poco estructuradas" y para el cual es conveniente que la evaluación del aprendizaje mida habilidades de razonamiento superior.

3. Jigsaw (Aronson, Stephan, Sikes, Blaney, \& Snapp, 1978). Es un método aplicable a variedad de materias y de especialización en una tarea. En el Jigsaw, los alumnos trabajan en equipos heterogéneos de 6 miembros. El profesor no da clases, pero se encarga de dividir el tema que quiere enseñar en seis partes, cada una de ellas única e imprescindible para comprender el tema, y de escribir el material de 
cada parte. La tarea es individual. Cada alumno recibe una parte del tema, "como una pieza de un puzzle jigsaw y ha de juntarla con las partes que tienen sus compañeros para aprender el cuadro completo" (Aronson, \& Bridgeman, 1979, p. 441). Para ello, el alumno debe estudiar individualmente su parte, discutirla dentro de su grupo de expertos y después enseñarla al resto de su equipo. La recompensa es individual en base a las notas de cada alumno en un examen sobre el tema completo.

4. Jigsaw II (Slavin, 1986). Los inconvenientes del método anterior, como son el coste para preparar el material de cada parte, la dificultad e, incluso, en determinados casos, la imposibilidad para dividir el tema o el que los alumnos se conviertan en expertos de una sola parte del tema, son superados por el Jigsaw II, adaptación del Jigsaw hecha por Slavin (1986). En éste, los estudiantes trabajan en equipos de 4-5 personas. El profesor prepara el tema, ahora sí, pudiendo cortar textos, añadir información o escribir material nuevo, según necesite. La tarea es grupal. Todos los alumnos leen el tema completo, pero a cada miembro del equipo se le proporciona un subtema sobre el que debe ser experto. Los estudiantes discuten los subtemas en "grupos de expertos», y luego regresan a sus equipos para enseñar su parte. La recompensa, al contrario que en Jigsaw original, es grupal en base a la suma de las puntuaciones obtenidas por los miembros del equipo en un examen individual sobre el tema completo.

El Jigsaw se ha usado, especialmente, en historia, pero también en áreas de matemáticas y expresión escrita; y el Jigsaw II en ciencias. Ambos métodos, dado que el alumno tiene el rol de experto, ayudan al desarrollo de situaciones de igual estatus (Aronson, \& Bridgeman, 1979).

5. Equipos de Rendimiento (Student Teams-Achievement Division, STAD; Slavin, 1978a). Este método es aplicable a una variedad de áreas. Los alumnos son asignados a equipos heterogéneos de 4-5 miembros. Las funciones del profesor y las características de la tarea son similares a las descritas en el método de Johnson y Johnson (1994), con la diferencia de que cada alumno posee su material de trabajo. La recompensa es grupal, dependiendo del grado en que cada uno de los miembros del equipo haya mejorado su calificación respecto a la anterior calificación en un examen individual. De este modo, independientemente de que los alumnos hubieran recibido una puntuación alta o baja en su anterior examen, tienen la posibilidad de contribuir por igual a la puntuación de su equipo.

En sus orígenes se empleó en el área de lenguaje (i.e. puntuación, gramática), posteriormente, gracias a su flexibilidad, ha sido empleado en diversidad de áreas. 
6. Equipos Torneo (Teams-Games-Tournaments, TGT; DeVries, \& Edwards, 1973, 1974; DeVries, Edwards, \& Slavin, 1978). Este método es en esencia igual al STAD, con la diferencia de que el examen individual y la nota del equipo se substituyen por un sistema de torneos académicos. Estos consisten básicamente en responder a preguntas, escritas en fichas dentro de una caja, sobre la lección presentada por el profesor y trabajada por cada alumno en sus correspondientes equipos. Concretamente, cada estudiante juega en una mesa de torneo contra estudiantes de otros equipos, pero del mismo nivel, tomando como referencia una evaluación anterior. Aquél que gana más puntos dentro de una mesa recibe 6 puntos, el siguiente $4 \mathrm{y}$ el siguiente 2 . De tal modo que cada estudiante, sea del nivel que sea, tiene la oportunidad de contribuir con una puntuación máxima a la puntuación de su equipo. Para llevar a cabo los siguientes torneos, los tres estudiantes que han sacado mejores puntuaciones forman una mesa de torneo, los siguientes tres forman otra y así sucesivamente. La recompensa es grupal en base al número de puntos que cada miembro consigue en el torneo.

En sus orígenes esta técnica surgió en el área de las matemáticas, pero posteriormente, gracias a su flexibilidad, al igual que el STAD, ha sido empleada en diversas áreas como ortografía o ciencias.

7. Equipos de Enseñanza Individualizada Asistida (Team-Assisted Individualization, TAI; Slavin, Leavey et al., 1984). Es un método específico para enseñar matemáticas en los primeros ciclos. Los alumnos son divididos en equipos de 4-5 miembros, compuestos de parejas o tríos pertenecientes a grupos de enseñanza de distintos niveles de habilidad matemática, en base a una evaluación previa. Así, por ejemplo, un equipo puede estar formado por dos alumnos de bajo nivel y dos alumnos de alto nivel. El profesor enseña a cada grupo, en vez de a la clase entera, empleando una enseñanza directa de habilidades matemáticas. Mientras el profesor enseña a un grupo, el resto de los alumnos por parejas continúan trabajando en su material de trabajo adecuado a su nivel y grado, compuesto por distintas unidades. Cuando un alumno tiene dudas en un ejercicio, intenta resolverlas bien con su pareja o bien con los miembros de su equipo de otros niveles, antes de acudir al profesor. Así, éste dispone de más tiempo para enseñar en los grupos. La recompensa es grupal en base a la media de las puntuaciones obtenidas por los miembros del grupo en un examen individual y al número medio de unidades realizadas por el equipo cada semana.

Este método, que combina el uso de los equipos cooperativos con la enseñanza individualizada en matemáticas, surgió para ofrecer las ventajas de la enseñanza individualizada o programada (i.e., apropiado nivel de instrucción) y resolver sus dos principales problemas (i.e., 
la escasa enseñanza directa $y$, por tanto, de calidad, y la falta de incentivos).

8. Equipos Cooperativos Integrados para la Lectura y la Redacción (Cooperative Integrated Reading and Composition, CIRC; Stevens, Madden, Slavin, \& Farnish, 1987). Es un método específico para enseñar a leer y escribir en los últimos cursos de primaria. La formación de los equipos, las funciones del profesor y la tarea de los alumnos son similares a las del TAI, con la diferencia de que el profesor enseña habilidades específicas de comprensión lectora (i.e. identificación de ideas principales) y los estudiantes en sus equipos trabajan en una serie de actividades de lectura y escritura (i.e., leerse historias uno a otro o buscar palabras en el diccionario). La recompensa es grupal en base a la media de las puntuaciones obtenidas por los miembros del grupo en un examen individual, a la calidad de la composición escrita individual y a las actividades diarias.

Algunas de las características que comparten el CIRC y el TAI son las siguientes. Ambos siguen, básicamente, esta secuencia: instrucción del profesor a equipos pequeños y homogéneos, práctica en equipos heterogéneos, evaluaciones del equipo y examen. Los estudiantes no pasan al examen hasta que todos los miembros del equipo dan el visto bueno. Por último, dado que los estudiantes trabajan con materiales adecuados a su nivel de lectura y de matemáticas, respectivamente, tienen las mismas oportunidades de contribuir al éxito del equipo.

9. Estructuras de Controversia (Constructive Controversy; Johnson, \& Johnson, 1979; Johnson, \& Johnson, 1994). Es un método específico para estudiar temas controvertidos (i.e. legalización de las drogas). Los alumnos trabajan en grupos de 4 miembros, formados por dos parejas, una que tiene la consigna de estar «a favor» y la otra «en contra». El profesor no da clases, como en los métodos tradicionales. Sus funciones principales son preparar los materiales sobre las dos posturas y supervisar el trabajo de los equipos. La tarea es grupal. El equipo debe escribir un informe grupal que recoja de la mejor forma posible el pensamiento de todos los miembros. Para ello, primero, cada pareja trabaja sobre el material que posee y, posteriormente, comparte esa información con la otra pareja, exponiendo de forma oral los puntos relevantes o rebatiendo algunos razonamientos, entre otras formas. Después los alumnos cambian sus roles para trabajar la otra postura. Por último, el equipo entero llega a un consenso. La recompensa es grupal en base al rendimiento de cada miembro en un examen individual que exige del conocimiento de ambas posturas.

Este método exige, entre otras, habilidades de razonamiento superior (Johnson, \& Johnson, 1994), por lo que su uso tal vez sea limitado con alumnos muy jóvenes. 
Aunque no se describen de forma explícita en cada método, hay otros dos aspectos que todos comparten. Uno, el profesor tiene un papel constante en el desarrollo de comportamientos cooperativos. Éste, a través del modelado y del entrenamiento en técnicas concretas tanto antes como durante la aplicación del método, ha de promover el espíritu de equipo y la cohesión, factores que, por otro lado, aumentan la productividad de los grupos y reducen los conflictos entre los alumnos. Dos, las recompensas, tanto individuales como grupales, pueden ser de distintos tipos. Por ejemplo, diplomas para los equipos con mejores puntuaciones, fotos de los equipos "ganadores» en un lugar de honor $\mathrm{u}$ hojas informativas de las puntuaciones de cada equipo para colocar en la cartelera de clase (véase Slavin, 1990).

No queremos dejar esta descripción de métodos sin mencionar la posibilidad de incorporar al AC las recientes tecnologías de información y comunicación (TICs). Existen programas informáticos que permiten a los estudiantes trabajar juntos en tareas comunes (véase Susman, 1998 para una revisión sobre la eficacia de la instrucción cooperativa basada en el ordenador) y tecnologías como Internet y el correo electrónico (e-mail) que permiten la cooperación, superando las barreras físicas entre los estudiantes.

\section{ESTUDIOS DE REVISIÓN Y META-ANÁLISIS SOBRE LOS EFECTOS DEL AC Y LOS MECANISMOS RESPONSABLES}

Una gran cantidad de estudios primarios han permitido estimar la influencia de los métodos descritos aquí sobre variables cognitivas, afectivas y sociales, con tamaños del efecto considerables en comparación con otros métodos. De hecho, Cohen (1994) afirma que no es necesario ya asentar el $\mathrm{AC}$ pues es un «método de enseñanza legitimado que puede ayudar a los estudiantes a aprender" (p. 30). Ello no obsta para conocer los resultados de los estudios de revisión y de meta-análisis sobre los métodos de AC, con el fin de hacer un uso más fundado de éstos.

Al revisar los más de veinte estudios de revisión y de meta-análisis que existen sobre el AC, nos referiremos tan sólo a algunos para conocer los efectos y los mecanismos que los explican. A este conocimiento se puede llegar comparando los métodos de AC con métodos tradicionales, o bien comparando los métodos de AC entre sí (Slavin, 1995b). Son éstos últimos los más interesantes a la hora de determinar los elementos críticos del AC, aunque también los más escasos. Por otra parte, la mayoría de los estudios de revisión se han centrado en los efectos del AC sobre el rendimiento académico y menos en los efectos sobre las relaciones intergrupales e interétnicas. Este hecho se explica en parte porque, al menos en teoría, los mecanismos que explican los efectos positivos en las relaciones intergrupales son claros y se refieren a los criterios de contacto de Allport, anteriormente tratados. De 
hecho, Slavin y Cooper (1999) revisan los efectos sobre las relaciones interétnicas de métodos tales como el STAD, TGT, TAI, Jigsaw, Jigsaw II, Aprender Juntos y Grupo de Investigación y encuentran que en casi la totalidad de los estudios hay mejoras en las actitudes tanto de miembros de grupos mayoritarios como minoritarios, cuando las aplicaciones satisfacen tales criterios. De aquí en adelante, se hace referencia, pues, a los estudios que examinan principalmente el efecto sobre el rendimiento académico, como variable dependiente.

Efectos positivos del AC sobre el rendimiento académico. Desde los dos primeros estudios de revisión (Sharan, 1980; Slavin, 1980), pasando por el estudio de Johnson, Maruyama, Johnson, Nelson y Skon (1981), el de Johnson y Johnson (1989) y el de Slavin (1990), hasta el más reciente (Johnson et al., 2000), se concluye que los métodos de AC tienen efectos positivos en el rendimiento académico (y en otras variables como la productividad y las actitudes hacia el aprendizaje) en comparación con otros métodos de enseñanza tradicionales. En algunos de estos estudios, no obstante, unos métodos muestran mayores efectos que otros. Por ejemplo, el estudio de Newmann y Thompson (1987), uno de los pocos realizados en secundaria, revisa 27 trabajos de gran calidad y cinco métodos: STAD, TGT, Jigsaw, Aprender Juntos y Grupo de Investigación. Encuentran que el $68 \%$ de las 37 comparaciones realizadas mostraban que el $\mathrm{AC}$ aumenta más el rendimiento que otras formas de enseñanza tradicional. El método con resultados más consistentes fue el STAD, y el que menos el Jigsaw, quizá debido, según los autores, a su falta de elementos necesarios, como luego discutiremos al revisar el estudio de Slavin (1983). Además, aunque los estudios emplearon más el $\mathrm{AC}$ en ciencias, los mejores resultados se observaron en matemáticas y en lengua. También fueron mejores los resultados en $2^{\circ}$ y $3^{\circ}$ de secundaria, aunque la mayoría de los estudios se hicieron en $1^{\circ}$ de secundaria y algunos en bachiller. El meta-análisis más reciente (Johnson et al., 2000), realizado sobre 158 trabajos publicados entre 1970 y 1999, revela que los ocho métodos de AC analizados allí dieron lugar a un rendimiento mayor que los competitivos o individualistas. Concretamente, Aprender Juntos, Estructuras de Controversia, TGT y Grupo de Investigación son los métodos con mayor tamaño del efecto, aunque los otros cuatro (i.e., Jigsaw, STAD, TAI y CIRC) también dieron lugar a ganancias importantes en el rendimiento frente a los métodos tradicionales.

Mecanismos que explican los efectos positivos del AC sobre el rendimiento académico. Los estudios anteriores muestran los efectos positivos del AC en comparación con otras formas de enseñanza, pero no examinan las características críticas que explican estos efectos. Uno de los estudios de revisión que identifica los elementos del AC responsables de la mejora del rendimiento académico es el de Slavin (1983; véase también Slavin, 1991, 1997). Slavin examinó 46 estudios que emplearon métodos de AC en contraste con otros competitivos o individualistas, al menos durante 2 semanas, en aulas de primaria y de secundaria, comparando los tres tipos 
de estructuras de recompensas mencionadas en la Introducción (i.e., grupal basada en el rendimiento del grupo, grupal basada en el aprendizaje individual, e individual) y según la estructura de la tarea (i.e., especializada y estudio en grupo). En comparación con métodos tradicionales, el AC da lugar a un claro aumento del rendimiento cuando emplea una estructura de recompensa grupal basada en el aprendizaje individual, y un efecto nulo de los métodos de AC que emplean una recompensa grupal basada en el rendimiento del grupo junto a una tarea de estudio grupal. No basta, al parecer, con métodos de AC que incluyan recompensas grupales basadas en el rendimiento del grupo y en una tarea común que resuelven entre todos, sino que hace falta incluir también la responsabilidad individual ("accountability» en inglés), esto es, asegurar que cada individuo haga su mejor esfuerzo y que todos ayuden en este empeño.

La segunda categoría de estudios de revisión que interesa mencionar aquí son los que comparan métodos de AC entre sí. Todavía hoy estos estudios son escasos. Cohen (1994), una de las autoras que aborda esta cuestión, hace una aportación de interés decidiendo cambiar el foco de atención de aspectos como la recompensa y la responsabilidad individual, hacia la importancia de la interacción en el logro de los objetivos educativos y los factores que afectan a dicha interacción, especialmente el tipo de tarea. Cohen aclara que existen dos variables que afectan a la tarea. Una, si se trata de una tarea grupal que sólo puede ser acometida exitosamente en grupo, o bien se trata de una tarea que puede realizarse de forma individual. Dos, si se trata de una tarea que tiene respuestas directas y correctas, o, por el contrario, de una tarea que exige solucionar los llamados «problemas mal estructurados», donde la respuesta correcta es difícil de alcanzar, si es que existe. Cohen revisa estudios generalmente realizados en el aula de clase y sólo compara métodos cooperativos entre sí.

Para abreviar señalaremos sólo algunas de las conclusiones de mayor interés para comprender los mecanismos del AC. Primera, si el objetivo es que los estudiantes desarrollen el pensamiento conceptual, de alto nivel, parece preciso que el profesor esté presente ayudando y estimulando a los alumnos durante la realización de la tarea, dado que la investigación muestra que los estudiantes tienden a trabajar en el nivel más concreto (p. 7). Segunda, el efecto de la interdependencia tanto de objetivos como de recursos está mediada por la cantidad y tipo de interacción, puesto que la interdependencia por sí sola no asegura la participación en el grupo. A su vez, la interacción depende de la tarea y la recompensa grupal no siempre es necesaria, sobre todo si la tarea, es lo suficientemente difícil e interesante como para que sea necesaria la participación de todo el grupo para lograr un buen resultado (véase Slavin, 1995b para una postura contraria). Tercera, respecto a la tarea y a su estructuración, parece que los extremos se tocan, esto es, tanto la excesiva como la falta de estructuración dan lugar a que los estudiantes se queden en un nivel concreto y pobre de interacción, sin atreverse a pensar por sí mismos y a entrar en discusiones e intercambios más ricos (p. 22). Este hecho puede explicar que el Grupo de Investigación tenga mejores resultados cuando se evalúa a los estu- 
diantes con preguntas difíciles, que el STAD, cuyo éxito aparece en tareas que sólo exigen adquirir información y memorizar la exposición del profesor. Finalmente, Cohen afirma que los beneficios académicos ocurren para los estudiantes de bajo rendimiento con independencia de la dificultad de la tarea, mientras que para los de alto y medio rendimiento sólo ocurren si tienen la oportunidad de ofrecer explicaciones y si la tarea no es excesivamente difícil, pues, en este último caso, pueden adoptar explicaciones propias de etapas inferiores a su edad (p. 11). De hecho, Slavin (1995b) señala que la mayor crítica a los métodos de $\mathrm{AC}$ proviene de quienes trabajan con alumnos superdotados. Sin embargo, este autor no encuentra datos suficientes en la investigación realizada hasta hoy para dudar de los beneficios del AC en esta población.

Otro reciente estudio de revisión de Springer, Stanne y Donovan (1999) vuelve a centrarse en la comparación de investigaciones sobre los efectos del trabajo de pequeños grupos, ya sea en $\mathrm{AC}$ o en aprendizaje colaborativo. Revisan un total de 39 estudios relacionados con el aprendizaje en pequeños grupos de estudiantes universitarios de ciencias, matemáticas, ingeniería y tecnología. Uno de los resultados interesantes de este estudio fue que no hubo diferencias significativas en el rendimiento de los estudiantes que trabajaron en pequeños grupos en $\mathrm{AC}$, aprendizaje colaborativo o una mezcla de ambos. Esto lo interpretan como un apoyo a la conclusión de que cualquier método que mantenga a los estudiantes envueltos activamente en el aprendizaje puede tener efectos positivos. No obstante, agregan que es necesario seguir avanzando en la comprensión de los elementos que hacen efectivo el aprendizaje en pequeños grupos.

\section{CONCLUSIONES}

La literatura examinada en este trabajo pone de manifiesto lo señalado al inicio: el AC es una propuesta educativa con efectos claramente positivos en la esfera cognitiva, afectiva y social de los estudiantes que participan en esta forma de enseñanza. No obstante, los últimos estudios reflejan la necesidad de conocer con más exactitud los fundamentos, los efectos y los mecanismos concretos que explican tales efectos cuando se emplean estos métodos. Por tanto, la investigación debe continuar para lograr fundar la práctica educativa del AC en la mejor evidencia empírica posible.

Ahora bien, la evidencia empírica disponible hoy, tanto sobre el fundamento, como sobre los beneficios y las características del AC, es suficiente para animar al empleo de la misma. De hecho, no es una idea arriesgada (o injustificada) afirmar que el uso del AC es una asignatura pendiente en aulas españolas, y no es un deseo vano esperar que, en el futuro, los esfuerzos de formación y de orientación del profesorado tengan en cuenta las potencialidades de esta opción. 


\section{BIBLIOGRAFÍA}

ADAMS, D.M., \& HAMM, M.E. (1990). Cooperative learning. Critical thinking and collaboration across the curriculum. Springfield, IL: Charlen C. Thomas.

ALLPORT, G.W. (1962). La naturaleza del prejuicio. Buenos Aires: Universitaria de Buenos Aires. (Trabajo original publicado en 1954).

ARONSON, E., \& BRIDGEMAN, D. (1979). "Jigsaw groups and the desegregated classroom: In pursuit of common goals". Personality and Social Psychology Bulletin, 4, 438-446.

ARONSON, E., STEPHAN, C., SIKES, J., BLANEY, N., \& SNAPP, M. (1978). The jigsaw classroom. Beverly Hills, CA: Sage. Resumen obtenido de PsycINFO Database Record 1980, Abstract Number 1980-51351-000.

COHEN, E. (1994). "Restructuring the classroom: Conditions for productive small groups". Review of Educational Research, 64, 1-35.

CREEMERS, B.P.M. y SCHEERENS, J. (1997). "Developments in the educational effectiveness research programme». International Journal of Educational Research, 21, 125-140.

DEVRIES, D.L., \& EDWARDS, K.J. (1973). «Learning games and student teams: Their effects on classroom process». American Educational Research Journal, 4, 307-318. Resumen obtenido de PsycINFO Database Record 1974, Abstract Number 197407905-001.

DEVRIES, D.L., \& EDWARDS, K.J. (1974). "Students teams and learning games: Their effects on cross-race and cross-sex interaction". Journal of Educational Psychology, 66, 741-749.

DEVRIES, D.L., EDWARDS, K.J. \& SLAVIN, R.E. (1978). «Biracial learning teams and four field experiments using teams-games-tournament». Journal of Educational Psychology, $70,356-362$.
DOMINGO, J., \& GIRALDO, B. (2002). Segunda jornada sobre aprendizaje cooperativo. Barcelona: Universitat Politécnica de Catalunya.

GARCÍA, R., TRAVER, J.A., \& CANDELA, I. (2001). Aprendizaje cooperativo. Fundamentos, características y técnicas. Madrid: CCS e ICCE.

GOIKOETXEA, E. (1990). «Formas de intervención en el ámbito escolar que producen actitudes favorables hacia minorías étnicas. Referencia al caso gitano». En T. Calvo-Buezas (Dtor.), Minorías étnicas: Formas de intervención. Manuscrito no publicado.

GRANT, C.A., \& SLEETER, C.E. (1986). "Race, class, and gender in education research: An argument for integrative analysis». Review of Educational Research, 56, 195-211.

JOHNSON, D.W., \& JOHNSON, R.T. (1979). "Conflict in the classroom: Controversy and learning". Review of Educational Research, 49, 51-70.

JOHNSON, D.W., \& JOHNSON, R.T. (1989). Cooperation and competition: Theory and research. Edina, MN: Interaction Book Company.

JOHNSON, D.W., \& JOHNSON, R.T. (1994). Learning together and alone: Cooperative, competitive, and individualistic learning (4. ${ }^{\mathrm{a}}$ ed.). Needham Heights, MA: Allyn \& Bacon.

JOHNSON, D.W., JOHNSON, R.T., \& HOLUBEC, E.J. (1995). Los nuevos círculos de aprendizaje. Cooperación en el salón de clases y en la escuela. Alexandria, VA: Association for Supervision and Curriculum Development.

JOHNSON, D.W., JOHNSON, R.T., HOLUBEC, E.J., \& ROY, P. (1984). Circles of learning: Cooperation in the classroom. Alexandria, VA: Association for Supervision and Curriculum Development.

JOHNSON, D.W., JOHNSON, R.T., \& STANNE, M.B. (2000). Cooperative 
learning methods: A meta-analysis. Bajado el 8 de marzo de 2002 de http://www.clcrc.com/pages/cl-methods.html.

JOHNSON, D.W., MARUYAMA, G., JOHNSON, R., NELSON, D., \& SKON, L. (1981). "Effects of cooperative, competitive, and individual goal structure on achievement: A metaanalysis". Psychological Bulletin, 89, 47-62.

KHMELKOV, V.T., \& HALLINAN, M.T. (1999). "Organizational effects on race relations in schools". Journal of Social Issues, 55 , 627-645.

LOBATO, C. (1998). Aprendizaje cooperativo en matemáticas. Zarautz, Guipúzcoa: Universidad del País Vasco. MADDEN, N.A., \& SLAVIN, R.E. (1983). "Mainstreaming students with mild handicaps: Academic and social outcomes". Review of Educational Research, 53, 519-569.

NEWMANN, F., \& THOMPSON, J.A. (1987). Effects of cooperative learning on achievement in secondary schools: A summary of research. Madison, WI: University of Wisconsin-Madison, National Center on Effective Secondary Schools.

O'DONNELL, A.M. (1996). «Effects of explicit incentives on scripted and unscripted cooperation». Journal of Educational Psychology, 88, 74-86.

OVEJERO, A. (1990). El aprendizaje cooperativo. Una alternativa eficaz a la enseñanza tradicional. Barcelona: PPU.

SERRANO, J.M., GONZÁLEZ-HERRERO, M.E., \& MARTÍNEZ-ARTERO, M.C. (1997). El aprendizaje cooperativo en matemáticas. Un método de aprendizaje cooperativo-individualizado para la enseñanza de las matemáticas. Murcia: Universidad de Murcia.

SHARAN, S. (1980). "Cooperative learning in small groups: Recent methods and effects on achievement, attitudes, and ethnic relations". Review of Educational Research, 50, 241-272.
SHARAN, S. \& SHACHAR, C. (1988). Language and learning in the cooperative classroom. New York: SpringerVerlag.

SHARAN, S., \& SHARAN, Y. (1992). Expanding cooperative learning through group investigation. Nueva York: Teacher's College Press.

SLAVIN, R.E. (1978a). "Student teams and achievement divisions". Journal of Research and Development in Education, 12, 39-49.

SLAVIN, R.E. (1978b). "Student teams and comparison among equals: Effects on academic performance and student attitudes. Joumal of Educational Psychology, 70, 532-538.

SLAVIN, R.E. (1979). "Effects of biracial learning teams on cross-racial friendships". Journal of Educational Psychology, 71, 381-387.

SLAVIN, R.E. (1980). "Cooperative learning». Review of Educational Research, 50, 315-342.

SLAVIN, R.E. (1983). "When does cooperative learning increase student achievement?". Psychological Bulletin, 94, 429-445.

SLAVIN, R.E. (1984). "Component building: A strategy for research-based instructional improvement». Elementary School Journal, 84, 255-269.

SLAVIN, R.E. (1986). Using student team learning ( $3 .^{\mathrm{a}}$ ed.). Baltimore, MD: John Hopkins University, Center for Research on Elementary and Middle Schools.

SLAVIN, R.E. (1987a). «A theory of school and classroom organization». Educational Psychologist, 22, 89-108.

SLAVIN, R.E. (1987b). “Grouping for instruction in the elementary school". Educational Psychologist, 22, 109127.

SLAVIN, R.E. (1990). Cooperative learning. Theory, research, and practice. Needham Heights, MA: Allyn and Bacon.

SLAVIN, R.E. (1991). "Group rewards make groupwork work". Educational Leadership, 48, 89-91. 
SLAVIN, R.E. (1995a). "A model of effective instruction». The Educational Forum, 59, 166-176.

SLAVIN, R.E. (1995b). Research on cooperative learning and achievement: What we know, what we need to know. Bajado el 25 Julio 2002, del sitio de la Web de Success for All Foundation: http:/www.successforall.net/resource/research/cooplearn.htm.

SLAVIN, R.E. (1997). "When does cooperative learning increase student achievement?». En E. Dubinsky, D. Mathews y B. E. Reynolds (Eds.), Readings in cooperative learning for undergraduate mathematics (pp. 7184). Washington, DC: The Mathematical Association of America. Resumen obtenido de PsycINFO Database Record 1997, Abstract Number 1997 09074-006.

SLAVIN, R.E., \& COOPER, R. (1999). "Improving intergroup relations: Lessons learned from cooperative learning programsm. Journal of Social Issues, 55, 647-663.

SLAVIN R.E., LEAVEY, M., \& MADDEN, N.A. (1984). "Combining cooperative learning and individualized instruction: Effects on student mathematics achievement, attitudes, and behaviors". Elementary School Journal, 84, 409-422.
SLAVIN, R.E., \& MADDEN, N.A. (1979). "School practices that improve race relations". American Educational Research Journal, 16, 169-180.

SLAVIN, R.E., MADDEN, N.A., \& LEAVEY, M. (1984). "Effects of team assisted individualization on the mathematics achievement of academically handicapped and nonhandicapped students». Journal of Educational Psychology, 76, 813-819.

SPRINGER, L., STANNE, M.E., \& DONOVAN, S.S. (1999). «Effects of small-group learning on undergraduates in Science, Mathematics, Engineering, and Technology: A meta-analysis». Review of Educational Research, 69, 21-51.

STEPHAN, W.G. (1978). "School desegregation: An evaluation of predictions made in Brown vs. Board of Education». Psychological Bulletin, 85, 217-238.

STEVENS, R., MADDEN, N., SLAVIN, R.E., \& FARNISH, A. (1987). "Cooperative integrated reading and composition: Two field experiments». Reading Research Quarterly, 22, 433-454.

SUSMAN, E.B. (1998). "Cooperative learning: A review of factors that increase the effectiveness of cooperative computer-based instruction». Journal of Educational Computing Research, 18, 303-332.

\section{RESUMEN}

El aprendizaje cooperativo (AC) es una de las formas de organizar la enseñanza con mayor respaldo teórico y empírico, y cada vez más empleada. Este trabajo revisa la teoría del contacto de Allport (1954/1962) para explicar por qué los métodos de AC tienen efectos positivos en las relaciones intergrupales e interétnicas. El modelo de Slavin (1984) se revisa para juzgar los elementos de una instrucción eficaz que logran ser satisfechos por el AC, dando lugar a efectos positivos en el rendimiento académico. Se describen, además, los nueve métodos de AC más investigados y se resumen los principales hallazgos sobre sus 
efectos, así como los mecanismos que explican dichos efectos, según los estudios de revisión y de meta-análisis.

Palabras clave: Aprendizaje cooperativo; colaborativo; aprendizaje en pequeños grupos; relaciones interétnicas; rendimiento.

\section{ABSTRACT}

Cooperative learning $(C L)$ is one potential form of classroom organization, witch is becoming more increasingly used among educators. This article revise Allport's contact theory (1954/1962) to explain why CL methods have positive effects on intergroup and interracial relationships. Slavin's (1984) model is revised in order to identify critical elements of effective instruction that become satisfy by $C L$ methods. In addition, the most researched $C L$ methods are described and the main findings about the CL effects and the mechanisms that explain them are described, based on the revision and meta-analysis studies.

Key words: Cooperative learning; collaborative learning; small-group learning; interracial relationships; achievement. 